\title{
POSTER
}

\section{Le syndrome KBG expression phénotypique et odontostomatologique : à propos d'un cas}

\section{Schulthess $A^{* 1}$, Guillerminet ${ }^{1}$, Ahossi $\mathbf{V}^{1}$, Denis $\mathrm{F}^{1,2}$}

1 - Service d'odontologie, CHU de Dijon

2 - EA 481 Neurosciences Intégratives et Cliniques

\section{Introduction}

Le syndrome KBG est une affection génétique rare (Prévalence 1/1 000 000) transmise selon le mode autosomique dominant. Le gène ANKRD11 (Ankyrin Repeat Domain 11) a été identifié en 2011 comme responsable de ce syndrome. Le syndrome KBG est caractérisé par une dysmorphie faciale particulière, des signes dentaires, des anomalies squelettiques et un retard de développement [1,2]. A partir d'un cas, nous avons précisé la description phénotypique de ce syndrome au travers des signes dentaires.

Les critères diagnostiques

Le syndrome KBG est généralement caractérisé par une dysmorphie faciale (visage triangulaire, implantation basse antérieure et postérieure des cheveux, synophris, hypertélorisme, ptose des paupières, une arête nasale large, un long philtrum), une macrodontie des incisives centrales supérieures, des anomalies squelettiques (une petite taille, anomalies costo-vertébrales) et une déficience intellectuelle légère à modérée.

\section{A propos d'un cas}

Une patiente atteinte d'un syndrome KBG (délétion interstitielle en 16q24.3 de 214 kb identifiée à l'origine de l'interruption du gène ANKRD11) a été adressée au service d'odontologie du CHU de Dijon pour préciser l'expression phénotypique du syndrome. Elle présente des troubles d'acquisition psychomotrice, une gibbosité dorsale, une syndactylie. On observe une implantation basse des cheveux, des fentes palpébrales courtes, des oreilles avec conque décollée et absence d'anthélix, une lèvre inférieure éversée et un palais haut et large. On note une macrodontie relative et transitoire des incisives centrales supérieures.

\section{Discussion}

Cette observation a permis de préciser le phénotype de cette patiente. En effet, un élément majeur du diagnostic est la macrodontie des incisives centrales supérieures. Dans notre cas, cette macrodontie est plus en rapport avec une dysharmonie dento-maxillaire qu'une dysharmonie dento-dentaire. Un décalage entre l'âge dentaire, l'âge osseux et l'âge civil est à prendre en considération afin de relativiser la macrodontie des incisives centrales décrite jusqu'à présent. Pour tous ces syndromes à expression faciale et bucco-dentaire l'expertise de l'odontostomatologiste est essentielle. 


\section{Conclusion}

Cette observation relève la variabilité phénotypique d'un syndrome. Elle contribue à enrichir les données évolutives de certaines anomalies chromosomiques rares en complément de l'analyse génétique.

Les auteurs ne déclarent aucun conflit d'intérêt.

\section{Références}

1-Herrmann J, Pallister PD, Tiddy W, Opitz JM : The KBG syndrome-syndrome of short stature, characteristic facies, mental retardation, macrodontia and skeletal anomalies. Birth. Defects. Orig. Artic. Ser. 1975; $11:$ 7-18.

2- Brancati F, Sarkozy A, Dallapiccola B. KBG syndrome. Orphanet J Rare Dis. 2006 12;1:50. 